\title{
Treatment effect variation in brain stimulation across psychiatric disorders
}

\author{
Stephanie Winkelbeiner ${ }^{1,2^{*}}$, Whitney Muscat ${ }^{3,4,5}$, Andrea Joanlanne ${ }^{3,4,5}$, Nikolaos Marousis ${ }^{1}$, Stefan \\ Vetter $^{1}$, Erich Seifritz ${ }^{1}$, Thomas Dierks ${ }^{2}, \&$ Philipp Homan ${ }^{1,3,4,5^{*}}$ \\ ${ }^{1}$ University Hospital of Psychiatry Zurich, Zurich, Switzerland. \\ ${ }^{2}$ University Hospital of Psychiatry and Psychotherapy, University of Bern, Bern, Switzerland. \\ ${ }^{3}$ Center for Psychiatric Neuroscience, Feinstein Institute for Medical Research, Manhasset, NY, USA. \\ ${ }^{4}$ Division of Psychiatry Research, Zucker Hillside Hospital, Northwell Health, New York, NY, USA. \\ ${ }^{5}$ Department of Psychiatry, Zucker School of Medicine at Northwell/Hofstra, Hempstead, NY, USA.
}

\begin{abstract}
Noninvasive brain stimulation methods such as transcranial magnetic stimulation (TMS) and transcranial direct current stimulation (tDCS) are promising add-on treatments for a number of psychiatric conditions. Yet, some of the initial excitement is wearing off. Randomized controlled trials (RCT) have found inconsistent results. This inconsistency is suspected to be the consequence of variation in treatment effects and solvable by identifying responders in RCTs and individualizing treatment. However, is there enough evidence from RCTs that patients do indeed respond differently to treatment? This question can be addressed by comparing the variability in the active stimulation group with the variability in the sham group across studies.

We searched MEDLINE/PubMed and included all double-blinded, sham-controlled RCTs and crossover trials that used TMS or tDCS in adults with a unipolar or bipolar depression, bipolar disorder, schizophrenia spectrum disorder, or obsessive compulsive disorder. In accordance with the PRISMA guidelines to ensure data quality and validity, we extracted a measure of variability of the primary outcome.

A total of 114 studies with 5005 patients were considered in the analysis. We calculated variance-weighted variability ratios for each comparison of active versus sham stimulation and entered them into a random-effects model. We hypothesized that treatment effect variation in TMS or tDCS would be reflected by increased variability after active compared with sham stimulation, or in other words, a variability ratio greater than one.

Across diagnoses, we found a slight increase in variability after active stimulation compared with sham (variability ratio $=1.05 ; 95 \% \mathrm{CI}, 1.01-1.11, \mathrm{P}=0.012$ ). This effect was likely driven by studies in patients with schizophrenia who received rTMS compared with sham (variability ratio $=1.11 ; 95 \% \mathrm{CI}, 1.03-1.2, \mathrm{P}=0.007)$.

In conclusion, this study found evidence for treatment effect variation in brain stimulation, particularly for studies in schizophrenia. The extent of this variation, however, was modest, suggesting that the need for personalized or stratified medicine is still an open question.
\end{abstract}

Keywords: heterogeneity in treatment response, noninvasive brain stimulation, transcranial magnetic stimulation, transcranial directcurrent stimulation, personalized treatment, unipolar and bipolar depression, schizophrenia spectrum disorder, bipolar disorder, obsessive-compulsive disorder

Correspondence concerning this article should be addressed to Stephanie Winkelbeiner, University Hospital of Psychiatry Zurich, Lenggstrasse 31, 8032 Zurich, Switzerland. E-mail: stephanie.winkelbeiner@bli.uzh.ch

\section{Introduction}

The emergence of noninvasive brain stimulation for the treatment of various psychiatric conditions has brought promising possibilities. Stimulation methods such as transcranial magnetic stimulation (TMS) and transcranial direct current stimulation (tDCS) have been used as addon treatment ${ }^{1}$ and have convinced with their minimal risk 
profile,$^{2}$ the prospect of noninvasively interfering with neuronal transmission, and the easy-to-use application. Popularity, particularly for TMS, increased considerably after the Food and Drug Administration's ${ }^{3}$ approval of its application as an add-on to the conventional therapy for major depressive disorder in 2008 and obsessive-compulsive disorder (OCD) in 2018.

Consistent evidence for the superiority of TMS or tDCS over sham for other psychiatric disorders is lacking so far. Positive symptoms in schizophrenia are one such example: the initially promising results ${ }^{4-6}$ developed into a more heterogeneous picture over the years. ${ }^{7}$ One explanation for this might be the drop in effect sizes over time. 8,9 Another reason, often brought up by both researchers and clinicians (including ourselves ${ }^{10}$ ), is to assume variation in treatment effects in brain stimulation. ${ }^{11}$ This assumption has motivated the idea to personalize medicine by identifying responders to treatment through predictive biomarkers. ${ }^{12-15}$

However, the question remains whether there is enough evidence to conclude that patients do indeed differ in their response to noninvasive brain stimulation. In addition, the extent of such variation would be important as well, as it would determine the corresponding need for personalized psychiatry. One way to address this question is by comparing the variance between treatment and control groups of randomized, controlled trials (RCT). ${ }^{16}$ Greater variability in the active stimulation group would indicate that there is a component of variation, the patient-by-treatment or subgroup-by-treatment interaction, indicating variability of treatment effects. ${ }^{17}$ This approach has recently been used in the context of antipsychotics ${ }^{18}$ and antidepressants. ${ }^{19-22}$ Perhaps surprisingly, most of these meta-analyses found little evidence for treatment effect variation. ${ }^{18-20,22}$

To evaluate the heterogeneity in treatment effects for noninvasive brain stimulation (TMS and tDCS), we used the same approach of examining variability ratios in RCTs with patients across psychiatric diagnoses (unipolar depression, schizophrenia specrtum disoder, OCD, and bipolar disorder). We hypothesized that (1) the often claimed treatment effect variation in brain stimulation would be indicated by increased variability after active stimulation compared with sham, reflected by an overall variability ratio (VR) of greater than one. Further, we hypothesized that (2) the variability ratio is not dependent on the diagnostic group or (3) stimulation method.

\section{Methods}

\section{Selection criteria}

We included all studies that met the following eligibility criteria: (1) study population of adult patients with either a diagnosis of unipolar or bipolar depression, schizophrenia spectrum disorder, OCD, or bipolar disorder; (2) using noninvasive brain stimulation including TMS and tDCS; (3) RCTs or crossover trials; (4) published in a peer-reviewed English journal; (5) no case reports, case series, opinion pieces; (6) no animal research.

\section{Search strategy}

We conducted a comprehensive search of the electronic database MEDLINE (PubMed.gov) from January 1999 - October 2018 with the following combination of keywords: "depression" or "MDD" or "unipolar" and "transcranial magnetic stimulation" or "rTMS" or "TMS" or "transcranial direct current stimulation" or "tDCS"; "bipolar" or "mania" and "transcranial magnetic stimulation" or "rTMS" or "TMS" or "transcranial direct current stimulation" or "tDCS"; "obsessive compulsive disorder" or "OCD" or "obsession" or "compulsion" and "transcranial magnetic stimulation" or "rTMS" or "TMS" or "transcranial direct current stimulation" or "tDCS"; "psychosis" or "schizophrenia” or "positive symptoms" or "auditory hallucinations" or "thought disorder" or "delusions" or "hallucinations" or "thinking" or "disorganization" and "transcranial magnetic stimulation" or "rTMS" or "TMS" or "transcranial direct current stimulation" or "tDCS". The only search filters that were applied were "human", and "English language". In addition to the electronic database search, we searched the references of recent reviews and meta-analyses. ${ }^{23-27}$

\section{Data extraction}

In adherence with the PRISMA guidelines, ${ }^{28}$ two independent researchers (A.J. and W.M.) conducted the literature search and screened the articles, eliminated duplicates, decided whether the article met the inclusion criteria, and extracted all relevant data from the final articles (Supplementary Figure 1). Their independent searches were compared by S.W., discrepancies investigated, and resolved by discussion. The risk of bias of the included studies was assessed independently by S.W. and N.M. according to the Cochrane Handbook for Systematic Reviews of Interventions. ${ }^{29}$

We extracted an available variance measure (standard deviation, standard error, or confidence interval) and means for the primary outcome measure at baseline and outcome for the active and sham group. Additionally, we extracted information on the study design, sample size, participants' characteristics (diagnosis, treated symptom, sex, and age), stimulation parameters (targeted hemisphere, number of sessions, and whether neuronavigation was used), and the effect size. For TMS studies, we extracted also target location and stimulation frequency. For tDCS studies, we extracted also electrode placement of the anode and cathode and stimulation intensity.

Note that in cases of multi-arm RCTs, we omited study arms that were not relevant to the question of this metaanalysis. ${ }^{30}$ For example, we omitted the arm of one study ${ }^{31}$ 
in which TMS as an add-on to sertralin was compared to sham, while we included the arm in which TMS as a standalone treatment was compared to sham.

\section{Data preprocessing}

The majority of the included studies were RCTs (87\%), with crossover trials making up only a small percentage (13\%). Nevertheless, the inclusion of crossover trials required additional considerations. ${ }^{30}$ We decided to exclude the second period of each crossover trial thus treating the trial as if it was a parallel trial. To check that this approach did not lead to biased results we conducted sensitivity analyses including also the second period of crossover trials (Supplementary Figure 2). Of note, variance measures and means had to be estimated from figures for 17 (12\%) studies. In the case of multi-arm trials, in which more than one arm of the RCT was considered relevant to this meta-analysis, ${ }^{31-49}$ we divided the sample size of the sham group by the number of intervention arms while retaining standard deviation $(s)$ and mean. ${ }^{30}$ This allowed us to create multiple pair-wise comparisons for those studies.

For the analysis, we used the pre-post difference scores to account for baseline differences. For the 121 (88\%) studies that reported raw outcome scores, we calculated the mean pre-post difference score $\left(M_{\Delta}\right)$ with

$$
\mathrm{M}_{\Delta}=\mathrm{M}_{\text {outcome }}-\mathrm{M}_{\text {baseline }}
$$

The calculation of the respective standard deviation $\left(s_{\Delta}\right)$ is less straightforward with ${ }^{50,51}$

$$
s_{\Delta}=\sqrt{s_{\text {active }}^{2}+s_{\text {sham }}^{2}-\left(2 \times \rho \times s_{\text {active }} \times s_{\text {sham }}\right)}
$$

and required an approximation for the overall correlation coefficient $(\rho)$. Thus, we used the $17(12 \%)$ studies that had reported the standard deviation for the active and sham groups for baseline $\left(t_{0}\right)$, outcome $\left(t_{1}\right)$, and pre-post difference scores to calculate the correlation coefficient for the active ( $\left.\rho_{\text {active }}\right)$ and sham $\left(\rho_{\text {sham }}\right)$ group, respectively, with

$$
\rho_{\text {active }}=\frac{s_{\text {active }_{t 0}}^{2}+s_{\text {active }_{t 1}}^{2}-s_{\text {active }_{\Delta}}^{2}}{2 \times s_{\text {active }_{t 0}} \times s_{\text {active }_{t 0}}}
$$

Next, we averaged over $\rho_{\text {active }}$ and $\rho_{\text {sham }}$ to obtain one correlation coefficient per comparison. Finally, we averaged across the 17 comparisons and used this average coefficient ( $\rho_{\text {average }}=0.57$ ) for imputation in the calculation of $s_{\Delta} \cdot{ }^{29,30}$ While this approach was possible for the majority of studies, there were 3 studies that had reported the standard deviation of the percentage pre-post difference scores ${ }^{52-54}$ and one that had reported the range of the pre-post difference scores ${ }^{55}$ but provided no information on the raw outcome scores. Therefore, those studies had to be excluded.

\section{Statistical analysis}

The variance of the active groups comprises the same variance components (within-patient variation, regression to the mean, and measurement error) as the sham group, with the only difference that the active group can comprise also a treatment-by-patient or treatment-by-subgroup interaction. This variance component reflects response differences between patients or subgroups. Thus, in the case of individual response difference, we would expect an increased variation $(\mathrm{VR}>1)$ in the active group compared with sham. To test this, we calculated the VR for each comparison of active stimulation (TMS or tDCS) versus sham as log variability ratio $(\ln V R)^{56}$ with

$$
\operatorname{lnVR}=\ln \left(\frac{s_{\text {active }}}{s_{\text {sham }}}\right)+\frac{1}{2\left(n_{\text {active }}-1\right)}-\frac{1}{2\left(n_{\text {sham }}-1\right)}
$$

where $s_{\text {active }}$ and $s_{\text {sham }}$ were the standard deviations of the pre-post difference scores, and $n_{\text {active }}$ and $n_{\text {sham }}$ the respective sample sizes. ${ }^{16}$ The corresponding sampling variance $\left(s_{\operatorname{lnVR}}^{2}\right)^{16}$ for each study was defined as

$$
s_{\text {lnVR }}^{2}=\frac{1}{2\left(n_{\text {active }}-1\right)}+\frac{1}{2\left(n_{\text {sham }}-1\right)}
$$

We weighted each $\operatorname{lnVR}$ with the inverse of its $s_{\operatorname{lnVR}}^{2}{ }^{57}$ To quantify the true individual response, after adjusting for within-patient variability and regression to the mean, ${ }^{17,58}$ we fitted a random-effects model stratified by diagnostic group. For better interpretability, we transformed the results back from the log scale: VR greater than 1 indicating greater variability under active stimulation compared with sham and a VR smaller than 1 indicating less variability under active stimulation compared with sham.

Finally, we calculated the standardized mean difference (SMD) as a general effect size measure to compare our analysis with previous reports. By taking the log of the ratio of means, the SMD was centered symmetrically around zero, yielding Hedges' g. ${ }^{59}$ We also plotted the SMDs against year of publication stratified by diagnostic group to examine the relationship of the effect sizes and time.

\section{Data and code availability}

All analyses were performed in R (version 3.5.2), ${ }^{60}$ the calculation of VR and SMD was done with the R package metafor (version 2.4.0). ${ }^{59}$ This paper was written using knitr (version 1.28$)^{61}$ in RStudio (version 1.2.5042). ${ }^{62}$ All data and code are freely available online to ensure reproducibility (https://osf.io/6w947/). This study was pre-registered on the Open Science Forum platform (https://osf.io/8uxec). 


\section{Results}

\section{Descriptive statistics}

A total of 114 were included that investigated treatment with active TMS or tDCS in depression, ${ }^{31,31,33,35,37-43,45-49,63-108}$ schizophrenia, ${ }^{5,6,32,34,44,109-134}$ OCD, ${ }^{36,135-145}$ and bipolar disorder ${ }^{40,146-151}$ compared with sham over the last 22 years. Some studies compared more than one active stimulation to sham which is why we considered 138 comparisons in this meta-analysis. We had to exclude studies that failed to report the necessary data to calculate pre-post difference scores (4 studies ${ }^{52-55}$ ) and that defined more than one primary outcome measure (1 study $\left.{ }^{152}\right)$. We excluded the second period of 12 crossover trials ${ }^{65,66,72,76,80,100,118,121,136,138,145,153}$ ) and dropped 1 study's $\operatorname{arm}^{31}$ that was irrelevant for this meta-analysis. Importantly, none of the included studies used a design such as a repeated crossover trial that would have allowed to estimate individual response directly. ${ }^{154}$

Overall, a total of 5005 patients were included. Of these $3240(65 \%)$ had a diagnosis of unipolar or bipolar depression, $1262(25 \%)$ schizophrenia spectrum disorder, 278 (6\%) OCD, and 224 (4\%) bipolar disorder. Further, 99 (87\%) comparisons investigated TMS and 15 (13\%) tDCS compared with sham. Our evaluation of the risk of bias showed that 61 (44\%) comparisons had a high risk of bias, while only $22(16 \%)$ a low risk. For $55(40 \%)$ comparisons the risk was unclear (see Supplementary Figure 3). Therefore, we conducted sensitivity analyses to investigate the reliability of our results (see Supplementary Figure 4). For more details on the included studies see Supplementary Figure 5 and the Supplementary Tables 1-14.

\section{Variability ratio}

We found an overall slightly increased variability after active stimulation compared with sham across diagnostic groups and stimulation methods (VR $=1.05$, 95\% CI: 1.01 , $1.1, \mathrm{P}=0.014$, Figure 1$)$. This effect was most likely driven by studies in patients with schizophrenia $(\mathrm{VR}=1.1,95 \%$ CI: $1.02,1.19, \mathrm{P}=0.014$, Figure 1$)$. The subgroup analyses by stimulation method showed that only for TMS studies in schizophrenia there was a $1 \%$ increase in variability evident after active compared with sham stimulation (Supplementary Figures 6-9). For all other diagnoses, there was no evidence for increased variability, irrespective of diagnostic group and stimulation method. We estimated that $1 \%$ of the total variance was due to heterogeneity in true effects and not attributable to sampling variance.

\section{Mean effect size}

Additionally, we calculated the standardized mean differences to obtain an index of the effectiveness of brain stimu-

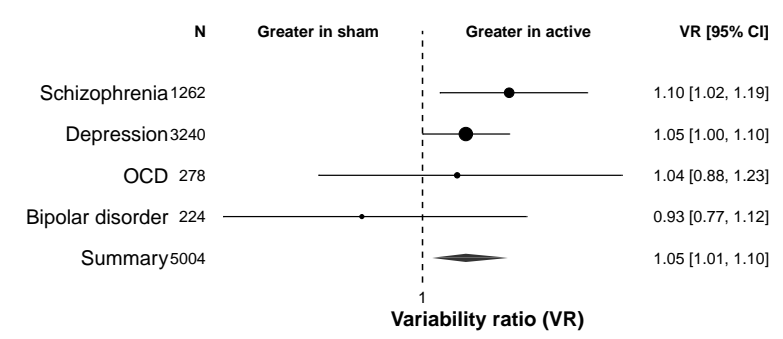

Figure 1. Variability ratio by disorder. The forest plot shows the VR together with its $95 \%$ confidence interval (CI) for active stimulation vs sham by disorder. Within the diagnostic groups, the variance was not significantly increased by the stimulation (TMS or tDCS) compared with sham, except for studies in patients on the schizophrenia spectrum.

lation. Overall, we found a medium to large effect size (SMD $=0.62,95 \% \mathrm{CI}: 0.32,0.92, \mathrm{P}<0.001$; Figure 2 ) across diagnostic groups. This indicates that noninvasive brain stimulation was on average more effective than sham and is in line with previous meta-analyses in depression (TMS: odds ratios between 1.69 and $7.44^{73,155}$; tDCS: odds ratio $=4.17^{155}$ ), schizophrenia (Hedges' $g$ between 0.39 and $0.63^{156,157}$ ), and OCD (Hedges' $g=2.94^{158}$ ).

Further, we found an inverse relationship of effect sizes and year of publication for the studies in patients with a schizophrenia spectrum diagnosis, but not for any of the other diagnostic groups (Supplementary Figure 10).

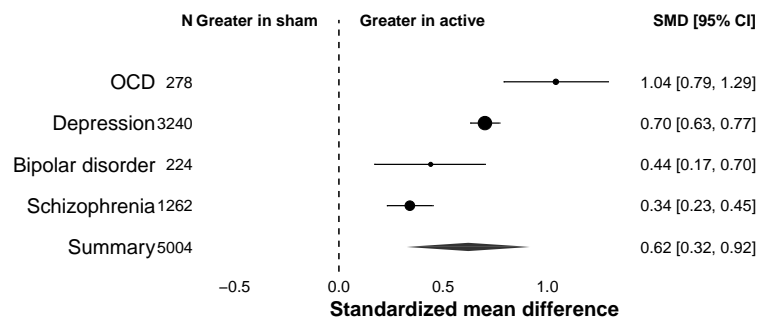

Figure 2. Standardized mean difference. The forest plot shows the standardized mean difference (SMD) together with its 95\% confidence interval (CI) for active stimulation vs sham by disorder. The overall effect size was greater for active stimulation compared with sham for all disorders.

\section{Discussion}

The observed variation in clinical trials and clinical practice is often viewed as proof that patients differ in their response to noninvasive brain stimulation. If this was true, one would expect that active stimulation should increase variability compared with sham stimulation. The empirical evidence in this study does indeed provide some support for this assumption, although our study could not determine whether this was due to differences between individuals or 
between subgroups, as both would lead to increased overall variability compared with sham. Yet, the size of this variability increase was modest and mostly driven by treatment effect variation in schizophrenia trials and to a smaller extent in depression trials. Consistent with previous metaanalyses, our study also found support for the overall efficacy of brain stimulation across disorders, even though a substantial risk of bias because of the mostly small studies has to be acknowledged.

Our findings lend at least some support for the recent trend in psychiatry to personalize treatment ${ }^{159,160}$ in general and brain stimulation in particular. ${ }^{12,161}$ One reason for this trend might be that the initally promising potential of noninvasive brain stimulation ${ }^{162}$ for the treatment of psychiatric and neurological conditions, ${ }^{24,25,27,73,155,157,158}$ has started to wear off. Despite medium to large effect sizes from depression trials, ${ }^{99,163}$ the promised beneficial effects did not appear to show in all patients. While some improved, others appeared to remain unchanged or even deteriorated. These oberved outcome differences leave patients and clinicans unsatisfied, and researchers tempted to assume variation in treatment effects. Naturally, this has motivated the search for biomarkers to identify those patients who are likely to respond more favorably to treatment and tailor treatments accordingly. Yet, despite encouraging attempts, ${ }^{12-14}$ (that occasionally did receive some pushback ${ }^{164,165}$ ) so far no reliable and clinically applicable biomarkers have been identified for treatment response prediction in individuals or subgroups.

One of the challenges in predicting treatment response might be rooted in the assumption that treatment effects vary in clinical trials and clinical practice. More often than not is this assumption the result of a somewhat arbitrary distinction between "responders" and "nonresponders". 166,167 Yet, the presence of other variance components that might explain a majority of the outcome variance is sometimes overlooked. ${ }^{168}$ Thus, evaluating variances can give a first hint about whether a personal or subgroup element of response is indeed present in the data. ${ }^{16}$ Our results provide some evidence for the presence of such treatment effect variation, driven mostly by studies in patients on the schizophrenia spectrum who were treated with TMS. For those studies, variability was slightly increased after TMS compared with sham. This deviates from similar recent meta-analyses of antipsychotic drug trials ${ }^{18,169}$ and antidepressant drug trials, ${ }^{19,20,22}$ where little evidence for treatment effect variation was found.

Notably, our results for the overall mean effect size of brain stimulation is largely in line with previous metaanalyses in depression (TMS: odds ratios between 1.69 and $7.44^{73,155}$; tDCS: odds ratio $=4.17^{155}$ ), schizophrenia (Hedges' $g$ between 0.39 and $0.63^{156,157}$ ), and OCD (Hedges' $\left.g=2.94^{158}\right)$. In addition, we found a negative correla- tion of effect size and publication year for schizophrenia studies, which was also in line with previous studies. ${ }^{8,9}$ This effect might be explained by the larger sample sizes ( $\mathrm{N}>70)$ of more recent studies, ${ }^{128,134}$ while the initial RCTs were performed in treatment groups of 10 or less patients (16 comparisons, ${ }^{41,42,45,65,66,80,82,131,132,138,141,142,145}$ $12 \%)$. Such small sample studies have low average statistical power, ${ }^{170,171}$ which can be consequential. Chances are lower to detect the "true" effect that actually reflects a statistically significant result. Thus, effect sizes are overestimated which reduces chances of reproducibility.

What are the clinical implications of our results for the treatment with noninvasive brain stimulation? This metaanalysis of variance differences from RCTs has provided some evidence to expect individual or subgroup differences in response to brain stimulation. One explanation might be the presence of subgroups that might have responded differently to brain stimulation with the more severely ill patients improving less or not at all and the less ill patients improving more. ${ }^{17}$ Such a case would argue for "stratified medicine" rather than "personalized medicine", in which subgroups of patients receive varying treatments. Thus, the analysis of variability ratios does not allow to distinguish between those cases, i.e. patient-by-treatment and subgroupby-treatment interactions. ${ }^{172}$ However, although we did find evidence for treatment effect variation, the overall extent of such variation was modest and most pronounced for schizophrenia, and even for schizophrenia the confidence intervall ranged from $2 \%$ to $19 \%$, indicating that the need for personalized or stratified medicine, respectively, could be anything from minimal to moderate. It is therefore an open question whether these findings are strong enough to warrant the search for predictive biomarkers, no matter how promising the approach. ${ }^{161,173,174}$

\section{Limitations}

Some limitations merit comment. First, we decided to calculate the variability ratio despite a significant mean-variance relationship for studies in patients on the schizophrenia spectrum (Supplementary Figure 11). This may limit the variability ratio in its applicability to identify shifts in variances and would call for the use of the coefficient of variation ratio (CVR) instead. ${ }^{16}$ Yet, the CVR is limited itself by the fact that the logarithm can only be taken from positive values. Thus, negative mean values cannot be dealt with without losing information. Using pre-post difference scores can result in negative values in the case of worsening of symptoms $(\bar{x}<0)$ or zero values in the case of no symptom change $(\bar{x}=0)$ from baseline to outcome. More importantly, the CVR is scale dependent. For values on the interval scales with a mean close to zero but a standard deviation far from zero, the CVR becomes arbitrarily large and the meanvariance proportionality which makes the CVR meaningful 
is lost. Therefore, the CVR should only be used for outcome data on the ratio scale with a true zero. ${ }^{175}$ Last, the majority of the included studies were rated as having high risk of bias, which suggests a low quality of evidence. This was due to various factors such as not clearly defined primary outcome measures, incomplete or selective data reporting, potentially misleading report of outcome data, and failure to report baseline data. We undertook various measure to account for these shortcomings (see Methods) and excluded the studies that did not report the values needed to infer the necessary values. In addition, we performed sensitivity analyses excluding the studies for which values had been inferred. The results of the sensitivity analyses showed the same direction as the main analyses.

\section{Conclusion}

Although this study did find evidence for treatment effect variation in brain stimulation (particularly for studies in schizophrenia), the extent of this variation was overall modest, suggesting that the need for personalized or stratified medicine is still an open question.

\section{Acknowledgments}

The authors thank Majnu John, PhD, Department of Mathematics, Zucker School of Medicine at Northwell/Hofstra, for advice on the analysis of the current study, as well as Anil Malhotra, MD, Department of Psychiatry, Zucker School of Medicine at Northwell/Hofstra, for helpful comments on the manuscript. These individuals received no additional compensation, outside of their usual salary, for their contributions.

\section{Conflict of interest}

SW, WM, AJ, NM, SV, ES, TD, and PH report no conflicts of interest.

\section{Funding/Support}

$\mathrm{PH}$ is supported by a NARSAD grant from the Brain \& Behavior Research Foundation (28445) and by a Research Grant from the Novartis Foundation (20A058).

\section{References}

1. Brunoni, A. R. et al. Noninvasive brain stimulation in psychiatric disorders: A primer. Brazilian Journal of Psychiatry 41, 70-81 (2019).

2. Rossi, S. et al. Safety, ethical considerations, and application guidelines for the use of transcranial magnetic stimulation in clinical practice and research. Clinical Neurophysiology 120, 2008-2039 (2009).

3. FDA. Class ii special controls guidance document: Repetitive transcranial magnetic stimulation (rTMS) systems guidance for industry and fda staff. (2011).
4. Hoffman, R. E. et al. Transcranial magnetic stimulation of left temporoparietal cortex in three patients reporting hallucinated voices. Biological Psychiatry 46, 130-132 (1999).

5. Hoffman, R. E. et al. Transcranial magnetic stimulation and auditory hallucinations in schizophrenia. The Lancet 355, 1073-1075 (2000).

6. Hoffman, R. E. et al. Transcranial magnetic stimulation of left temporoparietal cortex and medication-resistant auditory hallucinations. Archives of general psychiatry 60, 4956 (2003).

7. Marzouk, T., Winkelbeiner, S., Azizi, H., Malhotra, A. K. \& Homan, P. Transcranial magnetic stimulation for positive symptoms in schizophrenia: A systematic review. Neuropsychobiology 1-13 (2019).

8. Leucht, S. et al. Sixty years of placebo-controlled antipsychotic drug trials in acute schizophrenia: Systematic review, bayesian meta-analysis, and meta-regression of efficacy predictors. American fournal of Psychiatry 174, 927-942 (2017). 9. Slotema, C., Aleman, A., Daskalakis, Z. \& Sommer, I. Meta-analysis of repetitive transcranial magnetic stimulation in the treatment of auditory verbal hallucinations: Update and effects after one month. Schizophrenia research $\mathbf{1 4 2}$, 40-45 (2012).

10. Homan, P., Kindler, J., Hauf, M., Hubl, D. \& Dierks, T. Cerebral blood flow identifies responders to transcranial magnetic stimulation in auditory verbal hallucinations. Translational psychiatry 2, e189 (2012).

11. Guerra, A., López-Alonso, V., Cheeran, B. \& Suppa, A. Solutions for managing variability in non-invasive brain stimulation studies. Neuroscience letters (2017).

12. Drysdale, A. T. et al. Resting-state connectivity biomarkers define neurophysiological subtypes of depression. $\mathrm{Na}$ ture medicine 23, 28 (2017).

13. Pizzagalli, D. A. et al. Pretreatment rostral anterior cingulate cortex theta activity in relation to symptom improvement in depression. FAMA Psychiatry 75, 547 (2018).

14. Webb, C. A. et al. Rostral anterior cingulate cortex morphology predicts treatment response to internet-based cognitive behavioral therapy for depression. Biological Psychiatry: Cognitive Neuroscience and Neuroimaging 3, 255-262 (2018).

15. Rolle, C. E. et al. Cortical connectivity moderators of antidepressant vs placebo treatment response in major depressive disorder. FAMA Psychiatry 77, 397 (2020).

16. Nakagawa, S. et al. Meta-analysis of variation: Ecological and evolutionary applications and beyond. Methods Ecol Evol 6, 143-152 (2015).

17. Cortés, J. et al. Does evidence support the high expectations placed in precision medicine? A bibliographic review. F1000Research 7, (2018).

18. Winkelbeiner, S., Leucht, S., Kane, J. M. \& Homan, P. Evaluation of Differences in Individual Treatment Response in Schizophrenia Spectrum Disorders: A 
Meta-analysisEvaluation of Treatment Response Differences in SchizophreniaEvaluation of Treatment Response Differences in Schizophrenia. JAMA Psychiatry (2019) doi:10.1001/jamapsychiatry.2019.1530.

19. Munkholm, K., Winkelbeiner, S. \& Homan, P. Individual response to antidepressants for depression in adults - a simulation study and meta-analysis. (2019) doi:10.31219/osf.io/srzx5.

20. Plöderl, M. \& Hengartner, M. P. What are the chances for personalised treatment with antidepressants? Detection of patient-by-treatment interaction with a variance ratio metaanalysis. BMF open 9, (2019).

21. Maslej, M. M., Furukawa, T. A., Cipriani, A., Andrews, P. W. \& Mulsant, B. H. Individual differences in response to antidepressants: A meta-analysis of placebocontrolled randomized clinical trials. JAMA Psychiatry (2020) doi:10.1001/jamapsychiatry.2019.4815.

22. Volkmann, C. M. D., Volkmann, A. \& Mueller, C. On the treatment effect heterogeneity of antidepressants in major depression. A bayesian meta-analysis. medRxiv (2020).

23. Kubera, K. M., Barth, A., Hirjak, D., Thomann, P. A. \& Wolf, R. C. Noninvasive brain stimulation for the treatment of auditory verbal hallucinations in schizophrenia: Methods, effects and challenges. Frontiers in systems neuroscience 9, 131 (2015).

24. Lefaucheur, J.-P. et al. Evidence-based guidelines on the therapeutic use of repetitive transcranial magnetic stimulation (rTMS). Clinical Neurophysiology 125, 2150-2206 (2014).

25. Lefaucheur, J.-P. et al. Evidence-based guidelines on the therapeutic use of transcranial direct current stimulation (tDCS). Clinical Neurophysiology 128, 56-92 (2017).

26. Moseley, P., Alderson-Day, B., Ellison, A., Jardri, R. \& Fernyhough, C. Non-invasive brain stimulation and auditory verbal hallucinations: New techniques and future directions. Frontiers in neuroscience 9, 515 (2016).

27. Slotema, C. W., Blom, J. D., Lutterveld, R. van, Hoek, H. W. \& Sommer, I. E. Review of the efficacy of transcranial magnetic stimulation for auditory verbal hallucinations. $B i^{-}$ ological psychiatry 76, 101-110 (2014).

28. Moher, D. et al. Preferred reporting items for systematic review and meta-analysis protocols (prisma-p) 2015 statement. Systematic reviews 4, 1 (2015).

29. Higgins, J. P. \& Green, S. Cochrane handbook for systematic reviews of interventions. vol. 4 (John Wiley \& Sons, 2011).

30. Higgins, J. P., Eldridge, S. \& Li, T. Including variants on randomized trials. in Cochrane handbook for systematic reviews of interventions 569-593 (John Wiley \& Sons, Ltd, 2019). doi:10.1002/9781119536604.ch23.

31. Brunoni, A. R. et al. Heart rate variability is a trait marker of major depressive disorder: Evidence from the sertraline vs. Electric current therapy to treat depression clini- cal study. International fournal of Neuropsychopharmacology 16, 1937-1949 (2013).

32. Bais, L. et al. Short and long term effects of left and bilateral repetitive transcranial magnetic stimulation in schizophrenia patients with auditory verbal hallucinations: A randomized controlled trial. PloS one 9, e108828 (2014).

33. Blumberger, D. M. et al. A randomized doubleblind sham-controlled comparison of unilateral and bilateral repetitive transcranial magnetic stimulation for treatmentresistant major depression. The World Journal of Biological Psychiatry 13, 423-435 (2012).

34. Blumberger, D. M. et al. MRI-targeted repetitive transcranial magnetic stimulation of heschl?S gyrus for refractory auditory hallucinations. Brain stimulation 5, 577-585 (2012).

35. Blumberger, D. M. et al. Unilateral and bilateral mritargeted repetitive transcranial magnetic stimulation for treatment-resistant depression: A randomized controlled study. Journal of psychiatry \& neuroscience: IPN 41, E58 (2016).

36. Elbeh, K. A. M. et al. Repetitive transcranial magnetic stimulation in the treatment of obsessive-compulsive disorders: Double blind randomized clinical trial. Psychiatry Research 238, 264-269 (2016).

37. Fitzgerald, P. B. et al. A double blind randomized trial of unilateral left and bilateral prefrontal cortex transcranial magnetic stimulation in treatment resistant major depression. Journal of affective disorders 139, 193-198 (2012).

38. George, M. S. et al. A controlled trial of daily left prefrontal cortex tms for treating depression. Biological psychiatry 48, 962-970 (2000).

39. Höppner, J. et al. Antidepressant efficacy of two different rTMS procedures. European archives of psychiatry and clinical neuroscience 253, 103-109 (2003).

40. Loo, C. K. et al. International randomized-controlled trial of transcranial direct current stimulation in depression. Brain stimulation 11, 125-133 (2018).

41. Padberg, F. et al. Repetitive transcranial magnetic stimulation (rTMS) in pharmacotherapy-refractory major depression: Comparative study of fast, slow and sham rTMS. Psychiatry research 88, 163-171 (1999).

42. Paillère Martinot, M.-L. et al. Influence of prefrontal target region on the efficacy of repetitive transcranial magnetic stimulation in patients with medication-resistant depression: A [18F]-fluorodeoxyglucose pet and mri study. International Journal of Neuropsychopharmacology 13, 45-59 (2010).

43. Pallanti, S., Bernardi, S., Di Rollo, A., Antonini, S. \& Quercioli, L. Unilateral low frequency versus sequential bilateral repetitive transcranial magnetic stimulation: Is simpler better for treatment of resistant depression? Neuroscience 167, 323-328 (2010).

44. Slotema, C. W. et al. Can low-frequency repetitive 
transcranial magnetic stimulation really relieve medicationresistant auditory verbal hallucinations? Negative results from a large randomized controlled trial. Biological psychiatry 69, 450-456 (2011).

45. Speer, A. M., Wassermann, E. M., Benson, B. E., Herscovitch, P. \& Post, R. M. Antidepressant efficacy of high and low frequency rTMS at $110 \%$ of motor threshold versus sham stimulation over left prefrontal cortex. Brain stimulation 7 , 36-41 (2014).

46. Stern, W. M., Tormos, J. M., Press, D. Z., Pearlman, C. \& Pascual-Leone, A. Antidepressant effects of high and low frequency repetitive transcranial magnetic stimulation to the dorsolateral prefrontal cortex: A double-blind, randomized, placebo-controlled trial. The fournal of neuropsychiatry and clinical neurosciences 19, 179-186 (2007).

47. Su, T.-P., Huang, C.-C. \& Wei, I.-H. Add-on rTMS for medication-resistant depression: A randomized, doubleblind, sham-controlled trial in chinese patients. The fournal of clinical psychiatry 66, 930-937 (2005).

48. Theleritis, C. et al. Two versus one high-frequency repetitive transcranial magnetic stimulation session per day for treatment-resistant depression: A randomized shamcontrolled trial. The journal of ECT 33, 190-197 (2017).

49. Triggs, W. J. et al. Right and left dorsolateral prefrontal rTMS treatment of refractory depression: A randomized, sham-controlled trial. Psychiatry research 178, 467-474 (2010).

50. Abrams, K. R., Gillies, C. L. \& Lambert, P. C. Metaanalysis of heterogeneously reported trials assessing change from baseline. Statistics in medicine 24, 3823-3844 (2005).

51. Higgins, J. P., Green, S. \& others. Cochrane handbook for systematic reviews of interventions. (2008).

52. Boggio, P. S. et al. A randomized, double-blind clinical trial on the efficacy of cortical direct current stimulation for the treatment of major depression. International fournal of Neuropsychopharmacology 11, 249-254 (2008).

53. Fregni, F., Boggio, P. S., Nitsche, M. A., Rigonatti, S. P. \& Pascual-Leone, A. Cognitive effects of repeated sessions of transcranial direct current stimulation in patients with depression. Depression and anxiety 23, 482-484 (2006).

54. Schutter, D. J., Martin Laman, D., Honk, J. van, Vergouwen, A. C. \& Frank Koerselman, G. Partial clinical response to 2 weeks of $2 \mathrm{hz}$ repetitive transcranial magnetic stimulation to the right parietal cortex in depression. International Journal of Neuropsychopharmacology 12, 643-650 (2009).

55. Li, C.-T. et al. Efficacy of prefrontal theta-burst stimulation in refractory depression: A randomized shamcontrolled study. Brain 137, 2088-2098 (2014).

56. Hedges, L. V. \& Nowell, A. Sex differences in mental test scores, variability, and numbers of high-scoring individuals. Science 269, 41-45 (1995).

57. Viechtbauer, W. Conducting meta-analyses in $\mathrm{R}$ with the metafor package. Stat Software 36, 1-48 (2010).

58. Hecksteden, A. et al. Individual response to exercise training-a statistical perspective. Journal of Applied Physiology 118, 1450-1459 (2015).

59. Viechtbauer, W. \& Viechtbauer, M. Package metafor. The comprehensive $r$ archive network. Package metafor. (2017). 60. R Core Team. R: A language and environment for statistical computing. (R Foundation for Statistical Computing, 2018).

61. Knitr: A general-purpose package for dynamic report generation in $r$. (2020).

62. RStudio Team. RStudio: Integrated development environment for $r$. (RStudio, Inc., 2015).

63. Anderson, I. M. et al. Adjunctive fast repetitive transcranial magnetic stimulation in depression. The British fournal of Psychiatry 190, 533-534 (2007).

64. Avery, D. H. et al. A controlled study of repetitive transcranial magnetic stimulation in medication-resistant major depression. Biological psychiatry 59, 187-194 (2006).

65. Baeken, C. et al. Intensive hf-rTMS treatment in refractory medication-resistant unipolar depressed patients. fournal of affective disorders 151, 625-631 (2013).

66. Baeken, C. et al. The impact of accelerated hf-rTMS on the subgenual anterior cingulate cortex in refractory unipolar major depression: Insights from 18FDG pet brain imaging. Brain stimulation 8, 808-815 (2015).

67. Benadhira, R. et al. A randomized, sham-controlled study of maintenance rTMS for treatment-resistant depression (trd). Psychiatry research 258, 226-233 (2017).

68. Berman, R. M. et al. A randomized clinical trial of repetitive transcranial magnetic stimulation in the treatment of major depression. Biological psychiatry 47, 332-337 (2000). 69. Bortolomasi, M. et al. Long-lasting effects of high frequency repetitive transcranial magnetic stimulation in major depressed patients. Psychiatry research 150, 181-186 (2007).

70. Boutros, N. N. et al. Lack of a therapeutic effect of a 2-week sub-threshold transcranial magnetic stimulation course for treatment-resistant depression. Psychiatry research 113, 245-254 (2002).

71. Bretlau, L. et al. Repetitive transcranial magnetic stimulation (rTMS) in combination with escitalopram in patients with treatment-resistant major depression. A double-blind, randomised, sham-controlled trial. Pharmacopsychiatry 41, 41-47 (2008).

72. Brunoni, A. R. et al. Bifrontal tDCS prevents implicit learning acquisition in antidepressant-free patients with major depressive disorder. Progress in NeuroPsychopharmacology and Biological Psychiatry 43, 146-150 (2013).

73. Brunoni, A. R. et al. Trial of electrical direct-current therapy versus escitalopram for depression. New England fournal of Medicine 376, 2523-2533 (2017). 
74. Carpenter, L. L. et al. RTMS with a two-coil array: Safety and efficacy for treatment resistant major depressive disorder. Brain stimulation 10, 926-933 (2017).

75. Carretero, B. et al. Low-frequency transcranial magnetic stimulation in patients with fibromyalgia and major depression. Pain medicine 10, 748-753 (2009).

76. Fitzgerald, P. B. et al. Transcranial magnetic stimulation in the treatment of depression: A double-blind, placebocontrolled trial. Archives of general psychiatry 60, 1002-1008 (2003).

77. Fitzgerald, P. B. et al. A randomized, controlled trial of sequential bilateral repetitive transcranial magnetic stimulation for treatment-resistant depression. American fournal of Psychiatry 163, 88-94 (2006).

78. Garcia-Toro, M. et al. Modest adjunctive benefit with transcranial magnetic stimulation in medication-resistant depression. Fournal of Affective Disorders 64, 271-275 (2001). 79. Garcia-Toro, M. et al. Prefrontal repetitive transcranial magnetic stimulation as add on treatment in depression. Journal of Neurology, Neurosurgery \& Psychiatry 71, 546-548 (2001).

80. George, M. S. et al. Mood improvement following daily left prefrontal repetitive transcranial magnetic stimulation in patients with depression: A placebo-controlled crossover trial. American fournal of Psychiatry 154, 1752-1756 (1997). 81. Hausmann, A. et al. No benefit derived from repetitive transcranial magnetic stimulation in depression: A prospective, single centre, randomised, double blind, sham controlled ?Add on? Trial. Journal of Neurology, Neurosurgery \& Psychiatry 75, 320-322 (2004).

82. Holtzheimer III, P. E., Russo, J., Claypoole, K. H., RoyByrne, P. \& Avery, D. H. Shorter duration of depressive episode may predict response to repetitive transcranial magnetic stimulation. Depression and anxiety 19, 24-30 (2004). 83. Huang, M.-l. et al. Repetitive transcranial magnetic stimulation in combination with citalopram in young patients with first-episode major depressive disorder: A doubleblind, randomized, sham-controlled trial. Australian \& New Zealand Journal of Psychiatry 46, 257-264 (2012).

84. Januel, D. et al. A double-blind sham controlled study of right prefrontal repetitive transcranial magnetic stimulation (rTMS): Therapeutic and cognitive effect in medication free unipolar depression during 4 weeks. Progress in NeuroPsychopharmacology and Biological Psychiatry 30, 126-130 (2006).

85. Kang, J. I. et al. Frontostriatal connectivity changes in major depressive disorder after repetitive transcranial magnetic stimulation: A randomized sham-controlled study. The Journal of clinical psychiatry 77, e1137-e1143 (2016).

86. Klein, E. et al. Therapeutic efficacy of right prefrontal slow repetitive transcranial magnetic stimulation in major depression: A double-blind controlled study. Archives of general psychiatry 56, 315-320 (1999).
87. Koerselman, F., Laman, D. M., Willems, M. \& others. A 3month, follow-up, randomized, placebo-controlled study of repetitive transcranial magnetic stimulation in depression. The fournal of clinical psychiatry 65, 1323-1328 (2004).

88. Kreuzer, P. M. et al. Can repetitive transcranial magnetic stimulation prolong the antidepressant effects of sleep deprivation? Brain stimulation 5, 141-147 (2012).

89. Krstic, J. et al. Low-frequency repetitive transcranial magnetic stimulation in the right prefrontal cortex combined with partial sleep deprivation in treatment-resistant depression: A randomized sham-controlled trial. The journal of ECT 30, 325-331 (2014).

90. Leuchter, A. F. et al. Efficacy and safety of low-field synchronized transcranial magnetic stimulation (sTMS) for treatment of major depression. Brain stimulation 8, 787-794 (2015).

91. Loo, C. K. et al. A double-blind, sham-controlled trial of transcranial direct current stimulation for the treatment of depression. International fournal of Neuropsychopharmacology 13, 61-69 (2010).

92. Loo, C. K. et al. Transcranial direct current stimulation for depression: 3-week, randomised, sham-controlled trial. The British fournal of Psychiatry 200, 52-59 (2012).

93. Manes, F. et al. A controlled study of repetitive transcranial magnetic stimulation as a treatment of depression in the elderly. International Psychogeriatrics 13, 225-231 (2001).

94. Mantovani, A., Aly, M., Dagan, Y., Allart, A. \& Lisanby, S. H. Randomized sham controlled trial of repetitive transcranial magnetic stimulation to the dorsolateral prefrontal cortex for the treatment of panic disorder with comorbid major depression. Journal of affective disorders 144, 153-159 (2013).

95. Mogg, A. et al. A randomized controlled trial with 4month follow-up of adjunctive repetitive transcranial magnetic stimulation of the left prefrontal cortex for depression. Psychological Medicine 38, 323-333 (2008).

96. Möller, A. L., Hjaltason, O., Ivarsson, O. \& Stefánsson, S. B. The effects of repetitive transcranial magnetic stimulation on depressive symptoms and the p300 event-related potential. Nordic journal of psychiatry 60, 282-285 (2006).

97. Mosimann, U. P. et al. Repetitive transcranial magnetic stimulation: A putative add-on treatment for major depression in elderly patients. Psychiatry Research 126, 123-133 (2004).

98. Nongpiur, A., Sinha, V. K., Praharaj, S. K. \& Goyal, N. Theta-patterned, frequency-modulated priming stimulation enhances low-frequency, right prefrontal cortex repetitive transcranial magnetic stimulation (rTMS) in depression: A randomized, sham-controlled study. The fournal of neuropsychiatry and clinical neurosciences 23, 348-357 (2011).

99. O'Reardon, J. P. et al. Efficacy and safety of transcranial magnetic stimulation in the acute treatment of major depression: A multisite randomized controlled trial. Biological 
psychiatry 62, 1208-1216 (2007).

100. Palm, U. et al. Transcranial direct current stimulation in treatment resistant depression: A randomized doubleblind, placebo-controlled study. Brain stimulation 5, 242251 (2012).

101. Pascual-Leone, A., Rubio, B., Pallardó, F. \& Catalá, M. D. Rapid-rate transcranial magnetic stimulation of left dorsolateral prefrontal cortex in drug-resistant depression. The Lancet 348, 233-237 (1996).

102. Ray, S. et al. Efficacy of adjunctive high frequency repetitive transcranial magnetic stimulation of left prefrontal cortex in depression: A randomized sham controlled study. Journal of affective disorders 128, 153-159 (2011).

103. Rigonatti, S. P. et al. Transcranial direct stimulation and fluoxetine for the treatment of depression. European Psychiatry 23, 74-76 (2008).

104. Rossini, D., Lucca, A., Zanardi, R., Magri, L. \& Smeraldi, E. Transcranial magnetic stimulation in treatment-resistant depressed patients: A double-blind, placebo-controlled trial. Psychiatry Research 137, 1-10 (2005).

105. Rumi, D. O. et al. Transcranial magnetic stimulation accelerates the antidepressant effect of amitriptyline in severe depression: A double-blind placebo-controlled study. Biological psychiatry 57, 162-166 (2005).

106. Salehinejad, M. A., Ghanavai, E., Rostami, R. \& Nejati, V. Cognitive control dysfunction in emotion dysregulation and psychopathology of major depression (md): Evidence from transcranial brain stimulation of the dorsolateral prefrontal cortex (dlpfc). Journal of affective disorders 210, 241248 (2017).

107. Wang, Y.-M. et al. Randomized controlled trial of repetitive transcranial magnetic stimulation combined with paroxetine for the treatment of patients with first-episode major depressive disorder. Psychiatry Research 18 (2017).

108. Zheng, H. et al. High-frequency rTMS treatment increases left prefrontal myo-inositol in young patients with treatment-resistant depression. Progress in NeuroPsychopharmacology and Biological Psychiatry 34, 11891195 (2010).

109. Barr, M. S., Farzan, F., Tran, L. C., Fitzgerald, P. B. \& Daskalakis, Z. J. A randomized controlled trial of sequentially bilateral prefrontal cortex repetitive transcranial magnetic stimulation in the treatment of negative symptoms in schizophrenia. Brain stimulation 5, 337-346 (2012).

110. Brunelin, J. et al. Examining transcranial directcurrent stimulation (tDCS) as a treatment for hallucinations in schizophrenia. American fournal of Psychiatry 169, 719724 (2012).

111. Dlabac-de Lange, J. J. et al. Efficacy of bilateral repetitive transcranial magnetic stimulation for negative symptoms of schizophrenia: Results of a multicenter doubleblind randomized controlled trial. Psychological medicine 45, 1263-1275 (2015).
112. Fitzgerald, P. B. et al. A study of the effectiveness of bilateral transcranial magnetic stimulation in the treatment of the negative symptoms of schizophrenia. Brain Stimulation 1, 27-32 (2008).

113. Fröhlich, F. et al. Exploratory study of once-daily transcranial direct current stimulation (tDCS) as a treatment for auditory hallucinations in schizophrenia. European Psychiatry 33, 54-60 (2016).

114. Garg, S., Sinha, V. K., Tikka, S. K., Mishra, P. \& Goyal, N. The efficacy of cerebellar vermal deep high frequency (theta range) repetitive transcranial magnetic stimulation (rTMS) in schizophrenia: A randomized rater blind-sham controlled study. Psychiatry research 243, 413-420 (2016).

115. Hoffman, R. E. et al. Temporoparietal transcranial magnetic stimulation for auditory hallucinations: Safety, efficacy and moderators in a fifty patient sample. Biological psychiatry 58, 97-104 (2005).

116. Holi, M. M. et al. Left prefrontal repetitive transcranial magnetic stimulation in schizophrenia. Schizophrenia bulletin 30, 429-434 (2004).

117. Kimura, H. et al. A randomized, sham-controlled study of high frequency rTMS for auditory hallucination in schizophrenia. Psychiatry research 241, 190-194 (2016).

118. Klirova, M. et al. Individualized rTMS neuronavigated according to regional brain metabolism (18FGD pet) has better treatment effects on auditory hallucinations than standard positioning of rTMS: A double-blind, sham-controlled study. European archives of psychiatry and clinical neuroscience 263, 475-484 (2013).

119. Koops, S. et al. Theta burst transcranial magnetic stimulation for auditory verbal hallucinations: Negative findings from a double-blind-randomized trial. Schizophrenia bulletin 42, 250-257 (2015).

120. Li, Z. et al. Delayed effect of repetitive transcranial magnetic stimulation (rTMS) on negative symptoms of schizophrenia: Findings from a randomized controlled trial. Psychiatry research 240, 333-335 (2016).

121. Loo, C. K., Sainsbury, K., Mitchell, P., Hadzi-Pavlovic, D. \& Sachdev, P. S. A sham-controlled trial of left and right temporal rTMS for the treatment of auditory hallucinations. Psychological medicine 40, 541-546 (2010).

122. McIntosh, A. M. et al. Transcranial magnetic stimulation for auditory hallucinations in schizophrenia. Psychiatry Research 127, 9-17 (2004).

123. Mondino, M. et al. Effects of fronto-temporal transcranial direct current stimulation on auditory verbal hallucinations and resting-state functional connectivity of the left temporo-parietal junction in patients with schizophrenia. Schizophrenia bulletin 42, 318-326 (2015).

124. Paillère-Martinot, M.-L. et al. Active and placebo transcranial magnetic stimulation effects on external and internal auditory hallucinations of schizophrenia. Acta Psychiatrica Scandinavica 135, 228-238 (2017). 
125. Palm, U. et al. Prefrontal transcranial direct current stimulation for treatment of schizophrenia with predominant negative symptoms: A double-blind, sham-controlled proof-of-concept study. Schizophrenia bulletin 42, 12531261 (2016).

126. Poulet, E. et al. Repetitive transcranial magnetic stimulation does not potentiate antidepressant treatment. European Psychiatry 19, 382-383 (2004).

127. Prikryl, R. et al. A detailed analysis of the effect of repetitive transcranial magnetic stimulation on negative symptoms of schizophrenia: A double-blind trial. Schizophrenia research 149, 167-173 (2013).

128. Quan, W. X. et al. The effects of high-frequency repetitive transcranial magnetic stimulation (rTMS) on negative symptoms of schizophrenia and the follow-up study. Neuroscience letters 584, 197-201 (2015).

129. Rabany, L., Deutsch, L. \& Levkovitz, Y. Doubleblind, randomized sham controlled study of deep-tms addon treatment for negative symptoms and cognitive deficits in schizophrenia. Journal of Psychopharmacology 28, 686690 (2014).

130. Rollnik, J. D. et al. High frequency repetitive transcranial magnetic stimulation (rTMS) of the dorsolateral prefrontal cortex in schizophrenic patients. Neuroreport 11, 4013-4015 (2000).

131. Rosa, M. O. et al. Effects of repetitive transcranial magnetic stimulation on auditory hallucinations refractory to clozapine. The fournal of clinical psychiatry 68, 1528-1532 (2007).

132. Saba, G. et al. Transcranial magnetic stimulation in the treatment of schizophrenic symptoms: A double blind sham controlled study. Fournal of psychiatric research 40, 147-152 (2006).

133. Smith, R. C. et al. Effects of transcranial direct current stimulation on cognition, symptoms, and smoking in schizophrenia: A randomized controlled study. Schizophrenia research 168, 260-266 (2015).

134. Wobrock, T. et al. Left prefrontal high-frequency repetitive transcranial magnetic stimulation for the treatment of schizophrenia with predominant negative symptoms: A sham-controlled, randomized multicenter trial. Biological psychiatry 77, 979-988 (2015).

135. Gomes, P. V. O., Brasil-Neto, J. P., Allam, N. \& Rodrigues de Souza, E. A randomized, double-blind trial of repetitive transcranial magnetic stimulation in obsessive-compulsive disorder with three-month follow-up. The fournal of neuropsychiatry and clinical neurosciences 24, 437-443 (2012).

136. Haghighi, M. et al. Repetitive transcranial magnetic stimulation (rTMS) improves symptoms and reduces clinical illness in patients suffering from ocd-results from a singleblind, randomized clinical trial with sham cross-over condition. Journal of psychiatric research 68, 238-244 (2015).

137. Hawken, E. R. et al. Transcranial magnetic stimula- tion of the supplementary motor area in the treatment of obsessive-compulsive disorder: A multi-site study. International journal of molecular sciences 17, 420 (2016).

138. Jahangard, L. et al. Repetitive transcranial magnetic stimulation improved symptoms of obsessive-compulsive disorder, but also cognitive performance: Results from a randomized clinical trial with a cross-over design and sham condition. Neuropsychobiology 73, 224-232 (2016).

139. Kang, J. I., Kim, C.-H., Namkoong, K., Lee, C.-i. \& Kim, S. J. A randomized controlled study of sequentially applied repetitive transcranial magnetic stimulation in obsessivecompulsive disorder. The fournal of clinical psychiatry 70, 1645-1651 (2009).

140. Mansur, C. G. et al. Placebo effect after prefrontal magnetic stimulation in the treatment of resistant obsessivecompulsive disorder: A randomized controlled trial. International fournal of Neuropsychopharmacology 14, 13891397 (2011).

141. Mantovani, A., Simpson, H. B., Fallon, B. A., Rossi, S. \& Lisanby, S. H. Randomized sham-controlled trial of repetitive transcranial magnetic stimulation in treatmentresistant obsessive-compulsive disorder. International fournal of Neuropsychopharmacology 13, 217-227 (2010).

142. Mantovani, A. et al. Modulation of motor cortex excitability in obsessive-compulsive disorder: An exploratory study on the relations of neurophysiology measures with clinical outcome. Psychiatry research 210, 1026-1032 (2013). 143. Pelissolo, A. et al. Repetitive transcranial magnetic stimulation to supplementary motor area in refractory obsessive-compulsive disorder treatment: A shamcontrolled trial. International fournal of Neuropsychopharmacology 19, (2016).

144. Prasko, J. et al. The effect of repetitive transcranial magnetic stimulation (rTMS) on symptoms in obsessive compulsive disorder. A randomized, double blind, sham controlled study 332, (2006).

145. Shayganfard, M. et al. Repetitive transcranial magnetic stimulation improved symptoms of obsessive-compulsive disorders but not executive functions: Results from a randomized clinical trial with crossover design and sham condition. Neuropsychobiology 74, 115-124 (2016).

146. Dolberg, O., Dannon, P., Schreiber, S. \& Grunhaus, L. Transcranial magnetic stimulation in patients with bipolar depression: A double blind, controlled study. Bipolar disorders 4, 94-95 (2002).

147. Fitzgerald, P. B. et al. A negative double-blind controlled trial of sequential bilateral rTMS in the treatment of bipolar depression. Fournal of affective disorders 198, 158162 (2016).

148. Hernández-Ribas, R. et al. Identifying brain imaging correlates of clinical response to repetitive transcranial magnetic stimulation (rTMS) in major depression. Brain Stimulation 6, 54-61 (2013). 
149. Kaptsan, A., Yaroslavsky, Y., Applebaum, J., Belmaker, R. \& Grisaru, N. Right prefrontal tms versus sham treatment of mania: A controlled study. Bipolar Disorders 5, 36-39 (2003).

150. Praharaj, S. K., Ram, D. \& Arora, M. Efficacy of high frequency (rapid) suprathreshold repetitive transcranial magnetic stimulation of right prefrontal cortex in bipolar mania: A randomized sham controlled study. Fournal of affective disorders 117, 146-150 (2009).

151. Tavares, D. F. et al. Treatment of bipolar depression with deep tms: Results from a double-blind, randomized, parallel group, sham-controlled clinical trial. Neuropsychopharmacology 42, 2593 (2017).

152. Herwig, U. et al. Antidepressant effects of augmentative transcranial magnetic stimulation: Randomised multicentre trial. The British fournal of Psychiatry 191, 441-448 (2007).

153. Jandl, M. et al. Treating auditory hallucinations by transcranial magnetic stimulation: A randomized controlled cross-over trial. Neuropsychobiology 53, 63-69 (2006).

154. Senn, S. Mastering variation: Variance components and personalised medicine. Stat Med 35, 966-977 (2016).

155. Mutz, J., Edgcumbe, D. R., BrunoniMD, A. R. \& FuMD, C. H. Efficacy and acceptability of non-invasive brain stimulation for the treatment of adult unipolar and bipolar depression: A systematic review and meta-analysis of randomised sham-controlled trials. Neuroscience \& Biobehavioral Reviews (2018).

156. Slotema, C. W., Dirk Blom, J., Hoek, H. W. \& Sommer, I. E. Should we expand the toolbox of psychiatric treatment methods to include repetitive transcranial magnetic stimulation (rTMS)? A meta-analysis of the efficacy of rTMS in psychiatric disorders. Fournal of Clinical Psychiatry 71, 873 (2010).

157. Shi, C., Yu, X., Cheung, E. F., Shum, D. H. \& Chan, R. C. Revisiting the therapeutic effect of rTMS on negative symptoms in schizophrenia: A meta-analysis. Psychiatry research 215, 505-513 (2014).

158. Trevizol, A. P. et al. Transcranial magnetic stimulation for obsessive-compulsive disorder: An updated systematic review and meta-analysis. The journal of ECT 32, 262-266 (2016).

159. Ozomaro, U., Wahlestedt, C. \& Nemeroff, C. B. Personalized medicine in psychiatry: Problems and promises. $B M C$ medicine 11, 132 (2013).

160. Korte, S. M. et al. The many different faces of major depression: It is time for personalized medicine. European journal of pharmacology 753, 88-104 (2015).

161. Olbrich, S., Dinteren, R. van \& Arns, M. Personalized medicine: Review and perspectives of promising baseline eeg biomarkers in major depressive disorder and attention deficit hyperactivity disorder. Neuropsychobiology 72, 229240 (2015).
162. George, M. S. Whither tms: A one-trick pony or the beginning of a neuroscientific revolution. American fournal of Psychiatry 176, 904-910 (2019).

163. George, M. S. et al. Daily left prefrontal transcranial magnetic stimulation therapy for major depressive disorder: A sham-controlled randomized trial. Archives of general psychiatry 67, 507-516 (2010).

164. Dinga, R. et al. Evaluating the evidence for biotypes of depression: Methodological replication and extension of. NeuroImage: Clinical 22, 101796 (2019).

165. Mihalik, A., Adams, R. A. \& Huys, Q. Canonical correlation analysis for identifying biotypes of depression. Biological Psychiatry: Cognitive Neuroscience and Neuroimaging (2020) doi:10.1016/j.bpsc.2020.02.002.

166. Senn, S. Statistical pitfalls of personalized medicine. Nature (2018).

167. Homan, P. \& Kane, J. M. Clozapine as an early-stage treatment. Psychiatr Scand 138, 279-280 (2018).

168. Senn, S. Various varying variances: The challenge of nuisance parameters to the practising biostatistician. Statistical Methods in Medical Research 24, 403-419 (2015).

169. McCutcheon, R. A. et al. The efficacy and heterogeneity of antipsychotic response in schizophrenia: A metaanalysis. Molecular psychiatry 1-11 (2019).

170. Button, K. S. et al. Power failure: Why small sample size undermines the reliability of neuroscience. Nature Reviews Neuroscience 14, 365 (2013).

171. Amad, A. et al. Excess significance bias in repetitive transcranial magnetic stimulation literature for neuropsychiatric disorders. bioRxiv 614230 (2019).

172. Winkelbeiner, S. \& Homan, P. Is variance ratio a valid indicator of heterogeneous treatment effect?-reply. JAMA Psychiatry (2019) doi:10.1001/jamapsychiatry.2019.3382.

173. Cocchi, L. \& Zalesky, A. Personalized transcranial magnetic stimulation in psychiatry. Biological Psychiatry: Cognitive Neuroscience and Neuroimaging 3, 731-741 (2018).

174. Jog, M. V., Wang, D. J. \& Narr, K. L. A review of transcranial direct current stimulation (tDCS) for the individualized treatment of depressive symptoms. Personalized Medicine in Psychiatry (2019).

175. Mills, H. L. et al. Detecting heterogeneity of intervention effects using analysis and meta-analysis of differences in variance between arms of a trial. medRxiv (2020) doi:10.1101/2020.03.07.20032516. 